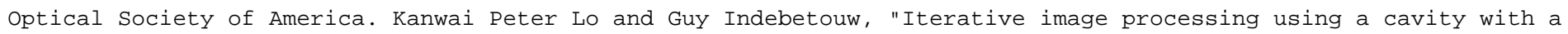
phase-conjugate mirror," Appl. Opt. 31, 1745-1753 (1992). doi: 10.1364/AO.31.001745

\title{
Iterative image processing using a cavity with a phase-conjugate mirror
}

\author{
Kanwai Peter Lo and Guy Indebetouw
}

\begin{abstract}
We describe an optical image processing system with regenerative feedback that utilizes a cavity with a phase-conjugate mirror. The system's characteristics and limitations are discussed, and its application to the implementation of iterative recovery algorithms (e.g., Gerchberg algorithm) is considered and demonstrated experimentally.
\end{abstract}

\section{Introduction}

Some of the most powerful techniques that are used to solve signal recovery and restoration problems as well as accomplish recognition and selection tasks make use of iterative algorithms. Several coherent, ${ }^{1-4}$ incoherent, ${ }^{5}$ and hybrid ${ }^{6}$ optical processors with feedback have been considered for implementing these algorithms optically. Many successful applications of these devices have been demonstrated in, e.g., inverse filtering, contrast control, edge enhancement, and analog computation.?

An optical implementation of iterative algorithms requires feedback and gain. Performances of passive, conventional feedback systems are limited by cavity losses and by the accumulation of phase error during each round trip. Several methods have been used to achieve optical gain. They include stimulated emission amplification ${ }^{1,8,9}$ and two-wave mixing in photorefractive materials. ${ }^{10-14}$ The problem of phase-error accumulation however, remains formidable, especially in devices with positive feedback that operate close to the threshold for self-oscillation.

Here we consider the alleviation of some of these problems by using a phase-conjugate mirror (PCM) in an optical feedback loop. Several feedback architectures that make use of a PCM have been proposed, e.g., for implementing a Wiener filter ${ }^{15,16}$ and providing for iterations in an optical associative memory. ${ }^{12,17}$ In principle, a PCM with gain can compensate for cavity losses and restore the phase of the wave front

The authors are with the Department of Physics, Virginia Polytechnic Institute and State University, Blacksburg, Virginia 24061.

Received 9 January 1991.

0003-6935/92/111745-09\$05.00/0.

(c) 1992 Optical Society of America. after each pair of round trips. It thus provides a regenerative feedback without accumulating phase errors. There is a drawback, however. The phasehealing property of the PCM, although necessary to avoid error accumulation, limits the class of operation that can be implemented in a PCM cavity. Our aim is to define these limitations and assess their consequences by means of examples.

In Section II we briefly review some general features of an optical feedback system. In Section III we describe the properties and limitations of a FabryPerot cavity with one PCM and stress the differences between such a cavity and a conventional passive feedback cavity. In Section IV the implementation of a restoration algorithm by using a PCM cavity is demonstrated. Some experimental results are shown and discussed in Section V.

\section{Positive versus Negative Feedback}

Both positive and negative feedbacks have been found to be useful in image processing. ${ }^{18,19}$ When estimating the differences between positive and negative feedback, it is easiest to discuss an idealized example of image restoration. Let us assume that a signal $E_{s}$ is spoiled by an optical system that is represented by an operator $\hat{S}_{s}$ and that the spoiled data $E_{i}$ are used as an input to an optical feedback system containing a forward operator $\hat{S}_{f}$ and a feedback operator $\hat{S}_{b}$. The relationships between $E_{s}, E_{i}$, and output $E_{o}$ of the feedback system are

$$
\begin{gathered}
E_{i}=\hat{S}_{s} E_{s}, \\
E_{o}=\hat{S}_{f} E_{i}+\alpha \hat{S}_{f} \hat{S}_{b} E_{o},
\end{gathered}
$$

where $\alpha$ is the feedback parameter.

We further assume (without justification at this stage) that these operators commute and that their 
eigenfunctions $\phi_{n}$ form a basis for a class of functions that includes $E_{s}, E_{i}$, and output $E_{o}$ of the feedback system. The validity of this assumption is discussed in Section III. We then have

$$
\begin{aligned}
E_{j} & =\sum_{n} e_{j n} \phi_{n}, \quad j=s, i, \text { or } o, \\
\hat{S}_{l} \phi_{n} & =\lambda_{l n} \phi_{n}, \quad l=s, f, \text { or } b .
\end{aligned}
$$

After substitution into Eqs. (1) and (2), we find that the relationship between the input and output coefficients is

$$
e_{o n}=\lambda_{n} e_{i n}=\lambda_{n} \lambda_{s n} e_{s n}
$$

where the eigenvalues $\lambda_{n}$ of the closed-loop system are given by

$$
\lambda_{n}=\frac{\lambda_{f n}}{1-\alpha \lambda_{f n} \lambda_{b n}}
$$

We examine Eqs. (5) and (6) and see that there are two possible ways of recovering $E_{s}$ from $E_{i}$ (i.e., $e_{o n} \approx e_{s n}$ ). The first way uses a feedback system where $\hat{S}_{f}=\hat{S}_{b}=\hat{S}_{s}$ (thus $\lambda_{f n}=\lambda_{b n}=\lambda_{s n}$ ), a negative feedback parameter $(\alpha<0)$, and a large gain $(|\alpha| \gg 1)$. Under these conditions, Eq. (6) reduces to approximately

$$
\lambda_{n} \sim\left(\alpha \lambda_{s n}\right)^{-1} .
$$

As is well known, one of the advantages of negative feedback is that it tends to reduce noise and cumulative errors. The large gain that is required, however, may lead to difficult stability problems. ${ }^{18}$

Recovery can also be achieved with positive feedback $(\alpha<0)$ if a complementary operator is used in the feedback loop: $\hat{S}_{b}=\hat{I}-\hat{S}_{s}$, while $\hat{S}_{f}=\hat{I}(\hat{I}$ is an identity operator). If the gain is close to unity $(\alpha \sim 1)$, Eq. (6) becomes

$$
\lambda_{n}=\left[1-\alpha\left(1-\lambda_{s n}\right)\right]^{-1} \sim \lambda_{s n}{ }^{-1} .
$$

Note that with positive feedback the gain must nearly compensate for the losses of the system. In other words, the system must be close to threshold. This may also cause some stability problems since a positive feedback system near threshold is sensitive to external perturbations.

In a conventional cavity with laser gain, the feedback parameter depends on the oscillation frequency and on the cavity length. For the cavity to be stable, it is sufficient that the feedback be negative at the frequency that experiences the maximum gain and that the net gain be smaller than that at the frequency, closest to maximum, for which positive feedback occurs. ${ }^{18}$

The situation is quite different for a PCM cavity in which the gain is provided by degenerate four-wave mixing in a photorefractive medium. As is shown in Section III, the feedback parameter at the pump frequency does not depend on the cavity length. If the net gain is $<1$, it is, in principle, possible to vary the phase of the feedback parameter by varying the phase of the pumps locally. With the most common geometry, however, the pumps have uniform phases over the entire PCM and, consequently, the feedback parameter is real and positive. Furthermore, with a gain larger than unity, the feedback is always positive and the cavity is unstable, thus preventing the implementation of negative feedback processing schemes.

There is actually another class of problems in which positive feedback is preferred. This class includes problems of selection and recognition. Here, instead of equalizing the eigenvalues, as is required for recovery, one needs to enhance the differences between eigenvalues until, for example, the preferred eigenfunction is unambiguously recognized. Such a selection could be achieved with positive feedback.

\section{Cavity with One PCM: Operator Theory}

\section{A. Steady-State Field Equations}

A Fabry-Perot cavity with one conventional mirror and a PCM is shown in Fig. 1 . Input $E_{i}^{\prime}$ is from the left, and output $E_{o}$ is the field transmitted by the cavity. The transmittance and the reflectance of the input mirror, which may include two-dimensional masks or filters, are represented by the operators $\mathscr{T}$ and $\mathscr{R}$, respectively. The PCM, which is assumed to be ideal, is represented by a reflectivity operator $\hat{\mu}$ :

$$
\hat{\mu}_{i}=r_{\mathrm{PCM} i} \widehat{p c}=\left|r_{\mathrm{PCM} i}\right| \exp \left(i \psi_{\mathrm{PCM} i}\right) \widehat{p c}, \quad i=1,2 .
$$

In this expression, $r_{\mathrm{PCM} i}$ is the steady-state complex amplitude reflectance of the PCM at the location $i=1$ or 2 , and $\psi_{\mathrm{PCM} i}$ is the phase term that is introduced by the PCM. The operator $\widehat{p c}$ denotes a phase-conjugation operator. The phase $\psi_{\mathrm{PCM} i}$ depends on the phaseconjugation mechanism. For example, in degenerate four-wave mixing with a photorefractive material (e.g., $\mathrm{BaTiO}_{3}$ ), the phase of the PCM reflectivity, under the no-absorption approximation and assuming a real coupling constant, is simply given by the

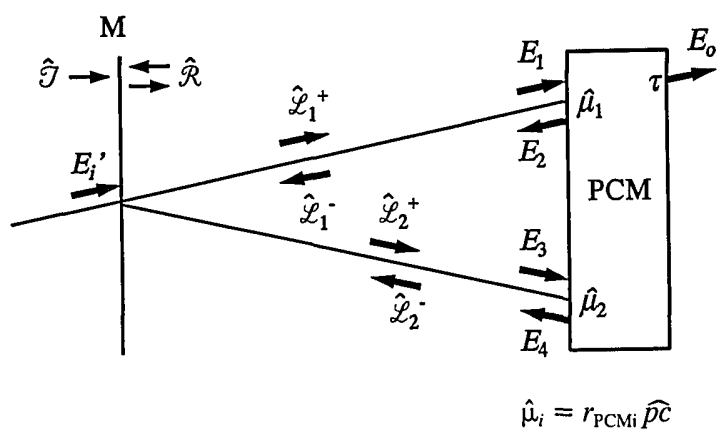

Fig. 1. Schematic diagram of a cavity with a PCM. The transmittance and reflectance of the mirror are represented by the operators $\hat{\mathscr{T}}$ and $\hat{\mathscr{R}}$, and the reflectivity of the PCM is characterized by the operators $\hat{\mu}_{i}$. The operators $\hat{l}_{1,2}^{ \pm}$describe the transfer of the optical field from the input mirror to the PCM and back along the primary and the secondary paths, respectively. 
sum of the phases of the pump beams ${ }^{20}$ :

$$
\psi_{\mathrm{PCMi}}=\phi_{1 i}+\phi_{2 i}
$$

We now derive an expression for the steady-state output of the cavity shown in Fig. 1. It is assumed that the field propagates in the cavity along two separate paths. The primary path contains the counter-propagating fields $E_{1}$ and $E_{2}$. The secondary path contains fields $E_{3}$ and $E_{4}$. These fields are defined at the entrance plane of the PCM.

Note that field $E_{1}$ results from the summation of field contributions that have been phase conjugated an even number of times, while field $E_{3}$ is the sum of contributions that have experienced an odd number of phase conjugations. As is discussed in Subsection III.B, the purpose of the two distinct paths is to allow for the spatial separation of these two fields.

The field transformations from the entrance mirror $\mathrm{M}$ to the PCM and back along the primary and secondary paths are described by the four linear operators $\mathscr{L}_{i}^{ \pm}, i=1,2$. These operators may include, e.g., free-space propagation, Fourier transformations, spatial masks, and spatial frequency filters. ${ }^{18,19}$

The steady-state fields satisfy the following boundary conditions:

$$
\begin{aligned}
& E_{1}=E_{i}+\hat{\mathscr{L}}_{1}^{+} \hat{\mathscr{R}} \hat{\mathscr{L}}_{2}^{-} E_{4}, \\
& E_{2}=\hat{\mu}_{1} E_{1}, \\
& E_{3}=\hat{\mathscr{L}}_{2}^{+} \hat{\mathscr{R}}_{1}^{-} \hat{\mathscr{L}}_{2}, \\
& E_{4}=\hat{\mu}_{2} E_{3},
\end{aligned}
$$

where $E_{i}$ is related to the input field $E_{i}{ }^{\prime}$, which is defined at input mirror $M$ by

$$
E_{i}=\hat{\mathscr{L}}_{1}+\hat{\mathscr{T}} E_{i}^{\prime} .
$$

After substituting into Eqs. (11), we obtain the following field equations for $E_{1}$ and $E_{3}$ :

$$
\begin{aligned}
& E_{1}=E_{i}+\hat{\mathscr{L}}_{1}^{+}+\hat{\mathscr{R}}_{2} \hat{\mathscr{L}}_{2}^{-} \hat{\mu}_{2} \hat{\mathscr{L}}_{2}^{+} \hat{\mathscr{R}} \hat{\mathscr{L}}_{1}^{-} \hat{\mu}_{1} E_{1}, \\
& E_{3}=\hat{\mathscr{L}}_{2}{ }^{+} \hat{\mathscr{R}} \hat{\mathscr{L}}_{1}^{-} \hat{\mu}_{1} E_{1} .
\end{aligned}
$$

If the output is taken as the field $E_{1}$ that is transmitted by the PCM at location 1, that is,

$$
E_{o}=\tau E_{1},
$$

where $\tau$ is the PCM transmittance, we find the steady-state output expression

$$
\begin{aligned}
& E_{o}=\tau E_{i}+\hat{\mathscr{L}}_{1}^{+} \hat{\mathscr{R}}_{\hat{\mathscr{L}}_{2}}^{-} \hat{\mu}_{2} \hat{\mathscr{L}}_{2}^{+} \hat{\mathscr{R}}_{\hat{\mathscr{L}}_{1}^{-}}^{-} \hat{\mu}_{1} E_{o} \\
& =\tau E_{i}+\beta \hat{\mathscr{O}} E_{o},
\end{aligned}
$$

where the operator $\hat{\mathscr{O}}$ is recognized as the feedbackloop operator, which consists of a double round trip in the cavity, and the feedback parameter $\beta$ is defined as

$$
\beta=|r|^{2} r_{\mathrm{PCM} 1}{ }^{*} r_{\mathrm{PCM} 2}=|r|^{2}\left|r_{\mathrm{PCM} 1}\right|\left|r_{\mathrm{PCM} 2}\right| \exp (i \psi) .
$$

Figure 2 shows a conventional block diagram of the feedback system that is described by Eq. (15).

If the input mirror $M$ is a conventional semitransparent mirror with transmission and reflection coefficients $t$ and $r$, respectively, Eq. (15) takes the simpler form

$$
E_{o}=\tau E_{i}+\beta \hat{\mathscr{L}}_{1}^{+} \hat{\mathscr{L}}_{2}^{-} \widehat{p c} \hat{\mathscr{L}}_{2}^{+} \hat{\mathscr{L}}_{1}^{-} \widehat{p c} E_{o}
$$

The phase $\psi$ of the feedback parameter is given by $\psi=$ $-\psi_{\mathrm{PCM} 1}+\psi_{\mathrm{PCM} 2}$, where $\psi_{\mathrm{PCM} 1}$ and $\psi_{\mathrm{PCM} 2}$ are the phases of PCM reflectance at location 1 (primary path) and location 2 (secondary path), respectively. This offers the possibility of controlling the phase of the feedback parameter through the phase of the pump beams at locations 1 and 2.

\section{B. Characteristics and Limitations}

In this section we point out three unique characteristics of the PCM cavity of Fig. 1 and discuss their consequences.

\section{Double-Path Feedback Loop}

The feedback-loop operator in Eq. (15) includes two round trips in the cavity. In the PCM cavity of Fig. 1, where the two paths are spatially separated, the output that is given by Eq. (14) includes only the field contributions that have been phase conjugated an even number of times. If the two paths overlap, the output must be taken as the superposition of fields $E_{1}$ and $E_{3}$. In general, these two fields interfere, and the output is not a simple function of the input.

An example is shown in Fig. 3. If the lens is one focal length away from the input mirror and from the PCM, the transfer operators are given by

$$
\hat{\mathscr{L}}^{+(-)}=\exp \left[i k L^{+(-)}\right] \hat{\mathscr{F}}
$$

where $\hat{\mathscr{F}}$ is a Fourier-transform operator and $L^{+(-)}$is the cavity optical length for the forward (backward) path.

After substitution from Eq. (18) into Eq. (13), using an input mirror with transmittance and reflectance $t$ and $r$, respectively, and using the fact that the operator $\hat{\mathscr{F}} \mathscr{F}$ is nothing but an inversion of coordinates, we find the cavity fields

$$
\begin{aligned}
& E_{1}(x, y)=E_{i}(x, y)+\beta E_{1}(x, y), \\
& E_{3}(x, y)=r r_{\mathrm{PCM} 1} \exp (i k L) E_{1}{ }^{*}(-x,-y),
\end{aligned}
$$

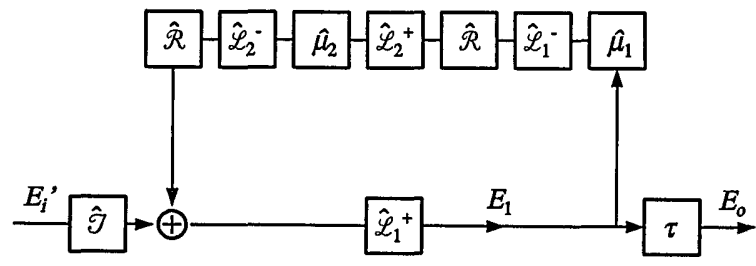

Fig. 2. Block diagram of the feedback cavity with the PCM shown in Fig. 1. 


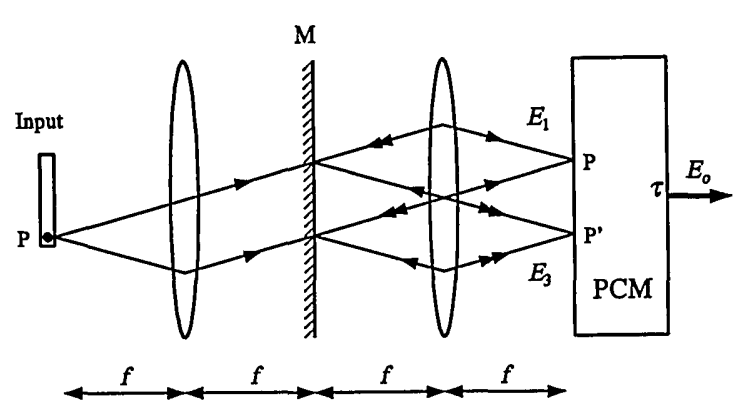

Fig. 3. Common path geometry of the Fabry-Perot cavity with a PCM. The output of the cavity is $E_{0}=\tau\left(E_{1}+E_{3}\right)$, and $E_{1}$ is proportional to the input while $E_{3}$ is proportional to the phase conjugate of the inverted input.

where $L=L^{+}+L^{-}$is the round-trip optical path length of the cavity and $\beta$ is as given by Eq. (16).

The output of the cavity is thus the sum of the cavity fields $E_{1}$ and $E_{3}$ :

$$
\begin{aligned}
E_{0}(x, y) & =\tau\left[E_{1}(x, y)+E_{3}(x, y)\right] \\
& =\frac{\tau}{1-\beta} E_{i}(x, y)+\frac{\tau r r_{\mathrm{PCM} 1} \exp (i k L)}{1-\beta^{*}} E_{i}^{*}(-x,-y) .
\end{aligned}
$$

It is seen that the output is a superposition of a field that is proportional to the input $E_{i}$ and a field that is proportional to the phase conjugate of a coordinate inverted input.

Note that this particularity of a PCM cavity output has been found to be useful for obtaining selfreferencing and inverting interferometers. ${ }^{21}$ It can also be used to produce holograms or to perform multiple transforms simultaneously. In image processing, however, this superposition of several fields at the output is in general not wanted. One way to avoid this problem is to provide for two distinct and spatially separated paths in the cavity, as was assumed to be the case in Subsection III.A (Fig. 1). Another way is to use an input that occupies only one quadrant of the input field and to synthesize a symmetrical cavity input that satisfies $E_{i}(x, y)=E_{i}^{*}(-x,-y)$.

\section{Feedback Parameter}

The second important characteristic of the PCM cavity is that the phase of the feedback parameter is determined by the phase difference of the PCM reflectance at two different locations [Eq. (17)], unlike that of a conventional cavity, which depends only on the cavity length.

In principle, it is possible to control the phase of the feedback parameter by controlling the phases of the pump beams at locations 1 and 2 (Fig. 1). In practice, however, it may not be easy to maintain this phase difference constant for long periods of time.

One possible geometry, which may make it easier to control the phase of the PCM reflectance independently for the two paths, is that of an unfolded cavity that uses two PCM's, as shown in Fig. 4. The steady-state output of this cavity is again given by Eq. (17). The only difference is that in the expression of

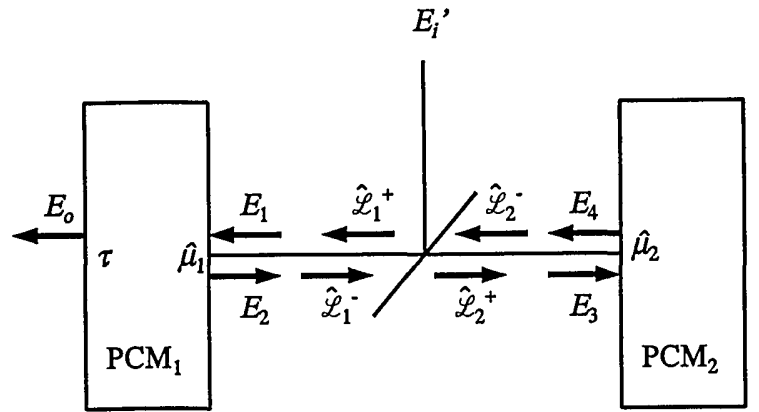

Fig. 4. Unfolded cavity with two PCM's, allowing for the independent control of the reflectivity $\hat{\mu}_{1}$ and $\hat{\mu}_{2}$ and thus for the control of the phase of the feedback parameter.

the feedback parameter the beam-splitter transmittance replaces the input mirror reflectance $r$.

If the two interaction regions in the single-PCM cavity share the same pump, which is the most common experimental situation, the feedback parameter is real positive since, in this case, $r_{\mathrm{PCM} 1}=r_{\mathrm{PCM} 2}$ once a steady state is reached. The cavity then always provides a positive feedback.

\section{Phase Cancellation}

The third characteristic of the PCM cavity that we discuss is a direct consequence of the phase-healing property of the PCM. As was already mentioned, this property is necessary to avoid phase-error accumulation in the cavity, but it restricts the kinds of operation that the system can perform.

For example, any phase-filtering operation will be canceled. To show this, let us assume that the transfer operators in both paths represent complex spatial-filtering operations, that is,

$$
\hat{\mathscr{L}}_{i}^{+(-)}=\exp \left[i k L_{i}^{+(-)}\right] \hat{\mathscr{F}} \hat{S}_{i} \hat{\mathscr{F}}, \quad i=1,2,
$$

where $L_{i}^{+(-)}$is the forward (backward) optical path length along path $i$ and $\hat{S}_{i}$ is an operator that represents the transmission of the field through a filter with a complex amplitude transmittance $t_{i}$. Below we define the operator $\hat{S}_{i}^{*}$ as representing the transmission through a filter with the complex conjugate transmittance $t_{i}^{*}$.

After substitution from Eq. (21) into Eq. (9), using the following relationships:

$$
\hat{\mathscr{F}} \widehat{p c}=\widehat{p c} \hat{\mathscr{F}}^{-1},
$$

$\hat{\mathscr{F}} \hat{\mathscr{F}} f(x, y)=f(-x,-y) \quad$ (coordinate inversion operator),

$$
\widehat{p c} \widehat{p c}=\hat{I} \quad \text { (identity operator) }
$$

$$
\hat{S}_{i} \widehat{p c}=\widehat{p c} \hat{S}_{i}^{*}
$$

we obtain the steady-state output of the cavity,

$$
E_{o}=\tau E_{i}+\beta \hat{\mathscr{F}} \hat{S}_{1} \hat{S}_{2} \hat{S}_{2}{ }^{*} \hat{S}_{1}^{*} \hat{\mathscr{F}}^{-1} E_{o}
$$

where $\mathscr{F}^{-1}$ is the inverse Fourier-transform operator. 
As defined, $\hat{S}_{1}$ and $\hat{S}_{2}$ obviously commute and have a common set of eigenfunctions $\left\{\phi_{k}\right\}$ with complex eigenvalues $\lambda_{i k}, i=1,2$ :

$$
\hat{\mathscr{F}} \hat{S}_{i} \hat{\mathscr{F}}^{-1} \phi_{k}=\lambda_{i k} \phi_{k} ; \quad i=1,2 .
$$

Assuming again that the eigenfunctions $\left\{\phi_{k}\right\}$ form a basis for $E_{i}$ and $E_{o}$, we can expand these fields as

$$
E_{i(0)}=\sum_{k} e_{i(0) k} \phi_{k}
$$

After substituting from Eq. (25) into Eq. (23) and comparing the coefficients of $\phi_{k}$, we obtain the relationship between the input and output coefficients:

$$
e_{o k}=\frac{1}{1-\beta\left|\lambda_{1 k}\right|^{2}\left|\lambda_{2 k}\right|^{2}} \tau e_{i k} \text {. }
$$

Clearly only the magnitude square of the eigenvalues of the filter operators $\hat{S}_{1}$ and $\hat{S}_{2}$ enter this transfer relationship. If they were phase-only filters, their effects would be perfectly canceled after each roundtrip pair.

It is interesting to contrast this result with some general conditions that the operators must satisfy to allow an expansion of the input and output in series of their eigenfunctions, something that has been assumed so far, without justification, to be valid.

Indeed, for the operator analysis to be valid, the eigenfunctions of the operators must be orthogonal and form a complete set. Furthermore, to be able to process more or less arbitrary inputs, the mode structure of the cavity must be rich enough to represent arbitrary spatially band-limited fieldamplitude distributions. One way of ensuring this in a conventional resonator is to require that the operators be self-adjoint Hilbert-Schmidt operators. ${ }^{18}$ This means that the transfer functions that are associated with these operators are Hermitian or, equivalently, that their point-spread functions (PSF's) are real. The PCM cavity, because of its phase-cancellation property, will automatically produce closed-loop PSF's that are real. It therefore seems that, to the extent that the transverse eigenmodes of the PCM cavity are degenerate, there is no restriction in the class of signals that can be processed. More realistically, the cavity can accommodate a broad class of suitably band-limited functions. Since the closed-loop operator is a self-adjoint Hilbert-Schmidt operator, the solution of Eq. (15) has the general form ${ }^{18}$

$$
E_{o}=\tau E_{i}+\sum_{n} \frac{\tau \lambda_{n}\left\langle E_{i}, \phi_{n}\right\rangle}{\lambda_{n}-\beta^{-1}} \phi_{n},
$$

where $\langle\cdot, \cdot\rangle$ indicates an inner product. It is assumed that $\beta^{-1}$ is not one of the eigenvalues of the operator o.

\section{Implementation of the Restoration Algorithm}

Many iterative restoration algorithms ${ }^{22-25}$ are based on amplitude-only filtering. They are thus not af- fected by the limitations that are discussed in Section III and should be realizable with a PCM cavity.

The Gerchberg algorithm, ${ }^{22}$ for example, needs only truncation operations in complementary (spatial and spatial frequency) domains for its implementation. The algorithm has been used for the extrapolation of noise-free band-limited functions and for the superresolution (spectral continuation) of functions that are defined in limited spatial domains. In Subsection IV.A, we review briefly the main features of the Gerchberg algorithm in order to provide a means of comparison with the operation of the PCM cavity that was used in the experiment.

\section{A. Gerchberg Algorithm}

The aim of the Gerchberg algorithm is to recover a function $f(x, y)$, which is defined in the spatial domain $D$ from band-limited data $g(x, y)$, the spatial spectrum of which vanishes outside the spatial frequency domain $B$.

We define the spatial and spatial frequency truncation operator $\hat{T}_{D}$ and $\hat{T}_{B}$ such that

$$
\begin{aligned}
& \hat{T}_{D} f(x, y)=\left\{\begin{array}{ll}
f(x, y), & \forall(x, y) \in D \\
0, & \text { otherwise }
\end{array},\right. \\
& \hat{T}_{B} \tilde{f}(\xi, \eta)=\left\{\begin{array}{ll}
\tilde{f}(\xi, \eta)=\hat{\mathscr{F}} f(x, y), & \forall(\xi, \eta) \in B \\
0, & \text { otherwise }
\end{array} .\right.
\end{aligned}
$$

The $m$ th estimate of $f(x, y)$ is obtained by adding the part of the spectrum that extends beyond the domain $B$ to the $(m-1)$ th estimate, according to the recursive relation

$$
g^{(m)}(x, y)=\hat{T}_{D}\left\{g(x, y)+\hat{\mathscr{F}}\left[\hat{I}-\hat{T}_{B}\right] \hat{\mathscr{F}}^{-1} \hat{T}_{D} g^{(m-1)}(x, y)\right\} .
$$

In this expression, $\left[\hat{I}-\hat{T}_{B}\right]$ is a complementary operator that is defined as

$$
\left[\hat{I}-\hat{T}_{B}\right] \tilde{g}(\xi, \eta)=\left\{\begin{array}{cc}
0, & \forall(\xi, \eta) \in B \\
\tilde{g}(\xi, \eta), & \forall(\xi, \eta) \notin B
\end{array}\right.
$$

and the first estimate is

$$
g^{(1)}(x, y)=\hat{T}_{D} g(x, y)=\hat{T}_{D} \hat{\mathscr{F}} \hat{T}_{B} \hat{\mathscr{F}}^{-1} f(x, y) .
$$

A comparison of Eq. (29) with Eq. (15) shows that this algorithm can be implemented with a PCM cavity with a round-trip operator:

$$
\begin{aligned}
\hat{\mathscr{O}} & =\hat{T}_{D} \hat{\mathscr{F}}\left[\hat{I}-\hat{T}_{B}\right] \hat{\mathscr{F}}^{-1} \hat{T}_{D} \\
& =[\hat{I}-\mathscr{G}] T_{D},
\end{aligned}
$$

where $\hat{\mathscr{G}}=\hat{T}_{D} \hat{\mathscr{F}} \hat{T}_{B} \hat{\mathscr{F}}^{-1}$.

If we assume that the eigenfunctions $\phi_{k}(x, y)$ of the operator $\hat{\mathscr{G}}$ are complete in the space of the functions with a limited domain $D$, one can substitute the expansion

$$
\hat{T}_{D} g^{(m)}=\sum_{e_{k}^{(m)}} \phi_{k}
$$


into Eq. (29) and obtain the recurrence formula for the expansion coefficients of the $m$ th Gerchberg estimate $^{25}$ :

$$
e_{k}^{(m)}=\frac{1-\left(1-\lambda_{k}\right)^{m}}{\lambda_{k}} e_{k}^{(1)}
$$

Before discussing the optical system that can be used to implement Eq. (29), two short remarks are in order.

The first is that in an optical implementation of the algorithm that is described by Eq. (29), it will not be possible to terminate the process after a prescribed number of iterations. In that sense, the feedback algorithm that is implemented in a cavity with a PCM is not, strictly speaking, iterative. When the number of iterations is large,

$$
e_{k}^{(m)}(m \rightarrow \infty) \rightarrow \frac{1}{\lambda_{k}} e_{k}^{(1)},
$$

which indicates that the final estimate is similar to the output of an inverse filter.

The second remark concerns the expansion in series of eigenfunctions. A special case, corresponding to simple rectangle functions for the truncation operators, has been extensively studied. In this case, the eigenfunctions are the prolate spheroidal wave functions. ${ }^{26}$ Even in this simple case, however, the eigenfunctions are tedious to calculate. The main advantage in trying to implement these algorithms optically is that the eigenfunctions need not be calculated or even known. With the appropriate operators in the cavity, the eigenfunctions will automatically be the cavity eigenmodes.

\section{B. Optical Implementation}

The optical system that is considered for implementing Eq. (29) is shown in Fig. 5. It is similar to the setup of Marks and Smith. ${ }^{27}$ The input data $g(x, y)$ is placed in the front focal plane of lens $L_{2}$. The input mirror of the cavity, which is placed in the back focal plane of $L_{1}$, has transparent openings that represent the spatial frequency truncation operator $\hat{T}_{B}$. The transmittance and reflectance operators of

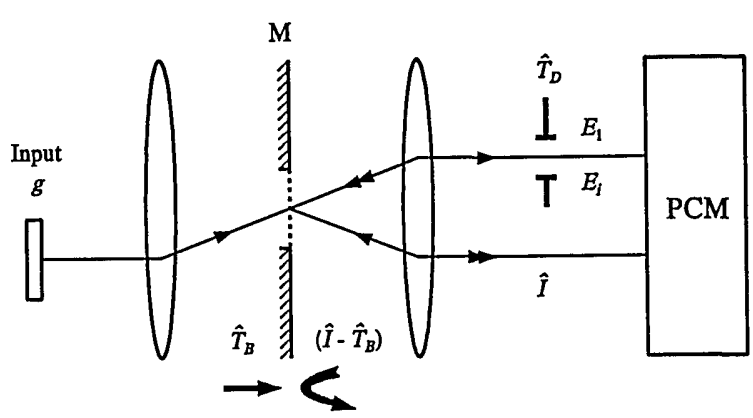

Fig. 5. Diagram of a setup for implementation of the Gerchberg algorithm. The input mirror implements the complementary spatial frequency truncation operator $\left[\hat{I}-\hat{T}_{B}\right]$. The primary path contains a spatial truncation operator $\hat{T}_{D}$ in plane $P$. The secondary path contains only an identity operator $\hat{I}$. that mirror are thus given by

$$
\hat{\mathscr{T}}=\hat{T}_{B}, \hat{\mathscr{R}}=r\left[\hat{I}-\hat{T}_{B}\right] .
$$

The output field equation is found to be

$$
E_{o}=\tau E_{i}+\beta \hat{T}_{D} \hat{\mathscr{F}}\left[\hat{I}-\hat{T}_{B}\right] \hat{\mathscr{F}} \widehat{p c} \hat{\mathscr{F}}\left[\hat{I}-\hat{T}_{B}\right] \hat{\mathscr{F}}_{T_{D}} \widehat{p c} E_{o},
$$

where

$$
E_{i}=\hat{T}_{D} \hat{\mathscr{F}} \hat{T}_{B} \hat{\mathscr{F}} g(x, y),
$$

and $\beta=|r|^{2} r_{\mathrm{PCM} 1} * r_{\mathrm{PCM} 2}$ as before.

If the relationships of Eqs. (22) are used together with the fact that $\hat{T}_{D}, \hat{T}_{B}$, and $\left[\hat{I}-\hat{T}_{B}\right]$ are projection operators that satisfy $\hat{P}^{2} \phi=\hat{P} \phi$ and $\hat{P} \widehat{p c}=\hat{p c} \hat{P}$, Eq. (37) takes the form

$$
E_{o}=\tau E_{i}+\beta \hat{T}_{D} \hat{F}\left[\hat{I}-\hat{T}_{B}\right] \hat{\mathscr{F}}^{-1} \hat{T}_{D} E_{o}
$$

which is equivalent to Eq. (29) if we let $g^{(m)}(m \rightarrow \infty) \rightarrow$ $E_{o}$.

After substitution from the expansions of Eq. (25) into Eq. (39), we find

$$
\begin{aligned}
e_{o k} & =\frac{1}{1-\beta\left(1-\lambda_{k}\right)} \tau e_{i k} \\
& \simeq \frac{1}{\lambda_{k}} \tau e_{i k} \quad(\beta \rightarrow 1)
\end{aligned}
$$

for the expansion coefficients of the output.

The output of the cavity approaches that of an inverse filter if the feedback parameter $\beta$ approaches one. Thus positive feedback is needed and the cavity must be close to threshold, as expected.

\section{Experimental Results}

\section{A. Experimental Setup}

For the experiment, a line input was used in the setup of Fig. 6. The input mirror had a transparent slit in it,

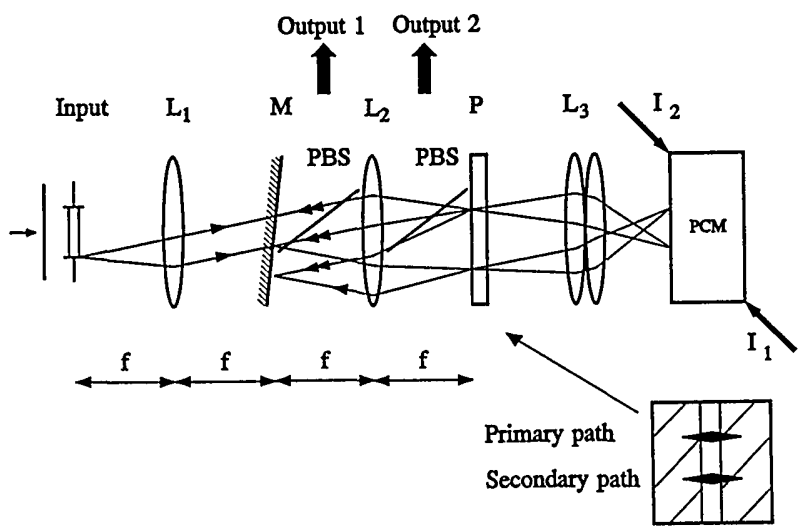

Fig. 6. Setup used for the experimental implementation of the Gerchberg algorithm. Phase conjugation is achieved by degenerate four-wave mixing in a single crystal of $\mathrm{BaTiO}_{3}$. The mirror $\mathrm{M}$ is slightly tilted to separate the primary and the secondary paths. Output ports 1 and 2 extract the extrapolated spatial frequency information and the enhanced spatial output, respectively. 
representing a low-pass filter $\hat{T}_{B}$. Another slit that was adjusted to the size of the data was used inside the cavity to represent the truncation $\hat{T}_{D}$. Since both the primary and the secondary paths pass through the slit, the secondary path contains an additional operator $\hat{T}_{D}$. This contrasts with Fig. 5, where only the primary path contains $\hat{T}_{D}$.

Since the opening that represents the truncation operator $\hat{T}_{D}$ is symmetrical, we have $\hat{\mathscr{F}}_{D} \hat{\mathscr{F}}^{-1}=$ $\hat{\mathscr{F}}^{-1} \hat{T}_{D} \hat{\mathscr{F}}$, and the output $E_{o}$ can be written as

$$
E_{o}=\tau E_{i}+\beta\left(\hat{T}_{D} \hat{\mathscr{F}}\left[\hat{I}-\hat{T}_{\mathrm{B}}\right] \hat{\mathscr{F}}^{-1}\right)^{2} E_{o},
$$

and the coefficients of its expansion are

$$
e_{o k}=\frac{1}{1-\beta\left(1-\lambda_{k}\right)^{2}} \tau e_{i k} .
$$

Since the PCM cavity commonly provides positive feedback, we have

$$
e_{o k} \simeq \frac{1}{\lambda_{k}\left(2-\lambda_{k}\right)} \tau e_{i k} \quad(\beta \rightarrow 1)
$$

It is interesting to note that the contributions of the eigenfunctions that are represented by large eigenvalues $\left(\lambda_{k} \simeq 1\right)$ are approximately the same as in an inverse filter, while the contributions of small eigenvalues $\left(\lambda_{k} \ll 1\right)$ are attenuated by a factor of 2 , compared with their contributions in an inverse filter. This could be interpreted as some kind of regularization.

\section{B. Experimental Results}

The PCM in the setup of Fig. 6 is an externally pumped single crystal of $\mathrm{BaTiO}_{3}(5 \mathrm{~mm} \times 5 \mathrm{~mm} \times 6$ $\mathrm{mm}$ ) in a degenerate four-wave mixing configuration. The laser was single-mode $\mathrm{Ar}^{+}$operating at $514 \mathrm{~nm}$. The angles between the pump beam and signal beam with the crystal normal were $47.5^{\circ}$ and $15.8^{\circ}$, respectively (outside the crystal, which was in air). Polarization was extraordinary for all the beams. The pump beams had powers of the order of $26 \mu \mathrm{W}$ for $P_{1}$ and 14 $\mu \mathrm{W}$ for $P_{2}$. The pump-beam diameters were $\sim 1 \mathrm{~mm}$ in the crystal. The signal beam was of the order of 20 $\mathrm{nW}$. With these parameters, a PCM reflectivity of the order of 1.7 was achieved. In the experiments, the reflectivity was adjusted to bring the cavity close to, but not above, threshold. This condition, of course, depends on the losses that are introduced by various intracavity elements.

Lenses $L_{1}$ and $L_{2}$ have $16-\mathrm{cm}$ focal lengths, providing a 1:1 imaging of the data onto a truncation slit that is $0.11 \mathrm{~mm}$ wide. The slit in the input mirror acts as a low-pass filter with a cutoff frequency of $\sim 7.4$ lines $/ \mathrm{mm}$. The truncation slit was reimaged onto the PCM with additional lenses.

The main reason for using a line input is to provide for two well-separated paths in the cavity while using a small interaction region in the crystal.

Input and restored output were photographed at the same location (output port 2 in Fig. 6) with the aid of a pellicle beam splitter (PBS). Output port 1 was used to observe the extrapolated spectrum on mirror $\mathrm{M}$.

Figure 7 shows some results for an input that consists of two sharp points. Both input and output data were recorded on the same film with about the same density and were processed simultaneously, ensuring that both data were recorded with the same contrast. Figure 7 shows an improvement of visibility of the two peaks by a factor of $\sim 30 \%$ (from a value of $\sim 0.6$ in the input to a value of $\sim 0.8$ in the output).

Figure 8 shows a trace through the extrapolated spectrum, which for a two-peak object should resemble a cosine function. Figure 8 shows that the parts of the extrapolated spectrum that are near the edges of the slit in the mirror are lost. This is because these edges are soft, thus providing little feedback in these regions of the mirror. Also, as expected, the extrapolation becomes increasingly noisy as one extends farther toward higher spatial frequencies.

\section{Discussion}

In analyzing the results of the previous section, one must keep in mind that the restoration algorithm that was discussed in Section III has severe fundamental limitations and that its implementation with an analog optical device such as that discussed in Section IV raises serious technical challenges.

The fundamental limitations come from the illposed nature of the problem. In trying to solve such problems with analog devices, one must face the fact that noise is unavoidable. While it is generally agreed that superresolution and analytic continuation are feasible with noise-free data, the extent to which it is feasible in the presence of noise is debatable. Some

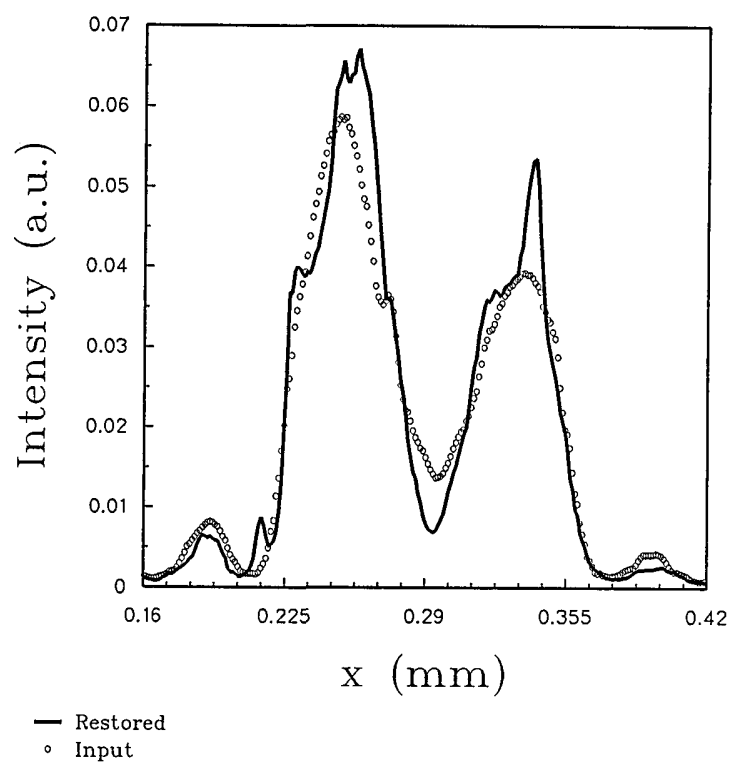

Fig. 7. Trace through the intensity distribution of the degraded image of a two-pixel input (dotted curve), and through that of the restored image (solid curve). The restored image shows a $30 \%$ improvement in visibility. 


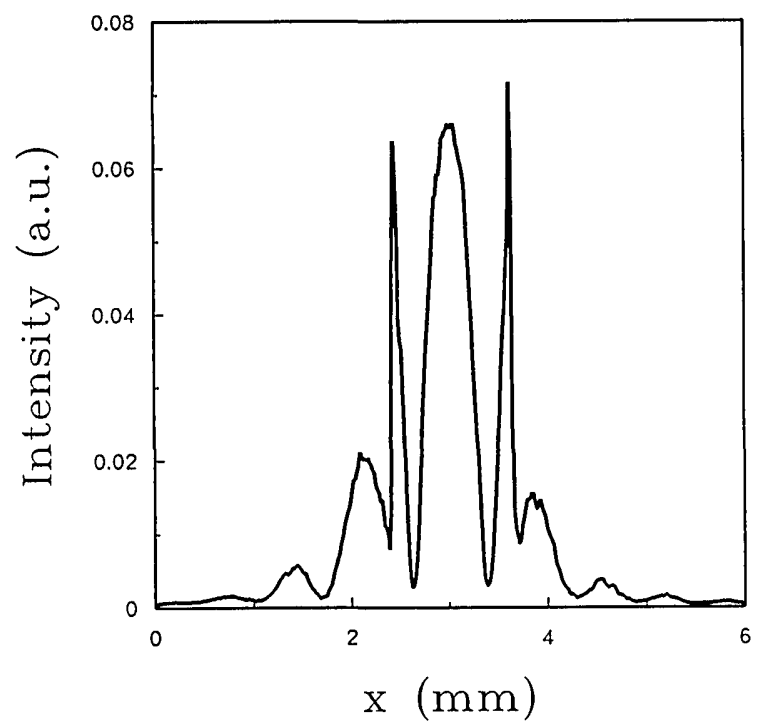

Fig. 8. Output at port 1 showing the extrapolated spatial frequency spectrum. The center portion is the input spectrum. The low intensity at the boundary of the band-limiting slit is due to the soft edges of the mirror, leading to low gain in these regions.

authors $\mathrm{s}^{28}$ would even claim that it is not physically realizable at all unless the data contain no more than a single pixel. In the case of a single pixel, of course, the problem is not one of resolution, but merely one of detection.

A more optimistic attitude is justifiable, but the expectations remain modest. For the experiment, we used a two-pixel object, the simplest possible object for which the concept of resolution is still meaningful. Experiments with more complicated objects did not lead to reproducible results. This may be due to some fundamental limitations or, more likely, to some technical difficulties such as determining the exact phase of the feedback parameter.

A number of experimental challenges had to be faced in the implementation that was described in Section V, the most important one being that one needs a cavity with positive feedback that operates close to threshold. This is the most unstable situation for an optical cavity. In our experimental setup, the fact that we used a cavity with photorefractive gain in $\mathrm{BaTiO}_{3}$ (a slow material and inherently difficult to control) enhanced these difficulties. They showed up in many different ways. For example, when the gain of the PCM is increased to bring the cavity closer to threshold, a critical slowdown is observed. More importantly, since the weaker parts of the signal distribution on the PCM have a higher gain, a large distortion may develop in the intensity distribution of the output.

For reasons that are probably tied to the above observations, it was also found that it was difficult to maintain the phase of the PCM reflectivity constant over a long period of time. If that phase changes differently for the two paths in the cavity, the phase of the feedback parameter changes, drastically altering the transfer function of the feedback system.
It was also observed that when the two paths (primary and secondary) share the same interaction region in the photorefractive crystal, a competition takes place. The beam in the primary path is usually much stronger than that in the secondary path, which contains the extrapolated data. Inherently, the weaker beam thus has a higher reflectivity. In the presence of the stronger beam, however, the reflectivity of the weaker one was observed to drop sizably. This drop of gain for the secondary path indirectly limits the amount of extrapolation achievable.

\section{Conclusion}

We have discussed critically the possibility of using a phase-conjugate mirror in an optical feedback system or cavity to implement iterative image processing algorithms. For such applications, one needs gain to overcome the cavity losses and phase healing to avoid the accumulation of aberrations and phase errors.

PCM cavities generally provide positive feedback. Negative feedback with a gain greater than one seems to be prohibited by the very nature of the PCM. The phase-healing property of a PCM was found to limit the class of algorithms that can be implemented with such devices. Many iterative recovery algorithms, however, are not affected by these limitations. More importantly, because many recovery problems are ill-posed and data are necessarily noisy, the chances of successfully solving these problems with analog devices should be weighted carefully. Some experiments were performed and their results seem to indicate that recovery by using Gerchberg-type algorithms is feasible optically but in a limited range only and for a class of objects that consists of a few pixels only. These limitations are to be expected from the nature of the problem.

Parts of this paper were presented at the 1990 OSA Annual Meeting in Boston. We thank the reviewers for their constructive comments.

\section{References}

1. R. V. Pole, H. Wieder, and E. S. Barrekette, "Reactive optical information processing. I: Theory of information recovery and resonator mode structure," Appl. Opt. 8, 1571-1575 (1967).

2. M. O. Hagler, "Active synthesis of inverse spatial filters," Appl. Opt. 10, 2783-2784 (1971).

3. D. P. Jablonowski and S. H. Lee, "A coherent optical feedback system for optical information processing," Appl. Phys. 8, 51-58 (1975).

4. E. Handler, and U. Roder, "Suppression of multiplicative disturbance by coherent optical feedback technique," Opt. Commun. 23, 352-356 (1977).

5. P. E. Kotljar, E. S. Nezhevenko, B. I. Spektor, and V. I. Feldbush, "Optical processing in feedback systems," in Optical Information Processing, E. S. Barrekette, G. W. Stroke, Y. E. Nesterikhin, and W. E. Kock, eds. (Plenum, New York, 1976), Vol. 2, pp. 155-170.

6. G. Hausler, and A. Lohmann, "Hybrid image processing with feedback," Opt. Commun. 21, 365-368 (1977).

7. J. N. Cederquist, "Optical feedback processing," in Optical Signal Processing, J. Horner ed. (Academic, New York, 1987), pp. 525-565. 
8. T. W. Hansch, F. Varsanyi, and A. L. Schawlow, "Image amplification with dye lasers," Appl. Phys. Lett. 18, 108-110 (1971).

9. R. P. Akins and S. H. Lee, "Coherent optical image amplification by an injection-locked dye amplifier at $632.8 \mathrm{~nm}$," Appl. Phys. Lett. 35, 660-663 (1979).

10. F. Laeri, T. Tschudi, and J. Albers, "Coherent cw image amplifier and oscillator using two-wave interaction in a $\mathrm{BaTiO}_{3}$ crystal," Opt. Commun. 47, 387-390 (1983).

11. P. Pellat-Finet and J. de Bougrenet de la Tocnaye, "Optical generator of spheroidal wave functions, using a BSO crystal," Opt. Commun. 55, 305-310 (1985).

12. D. Z. Anderson, "Coherent optical eigenstate memory," Opt. Lett. 11, 56-58 (1986).

13. B. H. Soffer, G. J. Dunning, Y. Owechko, and E. Marom, "Associative holographic memory with feedback using phaseconjugate mirrors," Opt. Lett. 11, 118-120 (1986).

14. H. Rajbenbach, Y. Fainman, and S. H. Lee, "Optical implementation of an iterative algorithm for matrix inversion," Appl. Opt. 26, 1024-1031 (1987).

15. O. Ikeda, T. Sato, and H. Kojima, "Analysis of a highresolution and large-dynamic-range spatial filter using a pair of facing phase conjugators with gains," J. Opt. Soc. Am. A $\mathbf{2}$, 1863-1868 (1985).

16. O. Ikeda, T. Sato, and H. Kojima, "Construction of a Wiener filter using a phase-conjugate filter," J. Opt. Soc. Am. A 3, 645-650 (1986).

17. D. Z. Anderson and M. C. Erie, "Resonator memories and optical novelty filter," Opt. Eng. 26, 434-444 (1987).

18. M. O. Hagler and S. V. Bell, "Nyquist stability conditions for laser feedback amplifiers with Gaussian or Lorentzian gain profiles," J. Opt. Soc. Am. A 3, 308-318 (1986).

19. P. De Santis, F. Gori, G. Guattari, and C. Palma, "Optical systems with feedback," Opt. Acta 23, 505-518 (1976).

20. M. Cronin-Golumb, B. Fischer, S. K. Kwong, J. O. White, and A. Yariv, "Nondegenerate optical oscillation in a resonator formed by two phase-conjugate mirrors," Opt. Lett. 10, 353355 (1985).

21. G. Indebetouw and K. P. Lo, "Real time moire interferometry using a Fabry-Perot cavity with a phase conjugate mirror," Appl. Opt. 28, 3893-3896 (1989).

22. R. W. Gerchberg, "Super-resolution through error-energy reduction," Opt. Acta 21, 709-720 (1974).

23. A. Papoulis, "A new algorithm in spectral analysis and bandlimited extrapolation," IEEE Trans. Circuits Syst. CAS-22, 735-742 (1975).

24. D. C. Youla, "Generalized image restoration by the method of alternating orthogonal projections," IEEE Trans. Circuits Syst. CAS-25, 694-702 (1978).

25. C. K. Rushforth and R. L. Frost, "Comparison of some algorithms for reconstructing space-limited images," J. Opt. Soc. Am. 70, 1539-1544 (1980).

26. D. Slepian, H. O. Pollak, and H. J. Landau, "Prolate spheroidal wave function,” Bell Syst. Tech. J. 40, 43-84 (1961).

27. R. J. Marks II and D. K. Smith, "Iterative coherent processor for bandlimited signal extrapolation," in International Optical Computing Conference I, W. T. Rhodes, ed., Proc. Soc. PhotoOpt. Instrum. Eng. 231, 106-111 (1980).

28. Y. A. Ananev and A. Y. Bekshaev, "More about the problem of superresolution in optics," Opt. Spectrosc. (USSR) 64, 139141 (1988). 